\title{
Chapter 7 \\ Whole Number Thinking, Learning and Development: Neuro-cognitive, Cognitive and Developmental Approaches
}

\author{
Joanne Mulligan (1), Lieven Verschaffel (1)

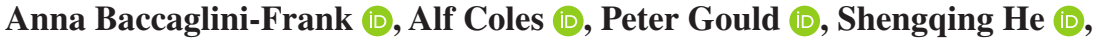 \\ Yunpeng Ma, Jasmina Milinković 마, Andreas Obersteiner (D), Nicole Roberts, \\ Nathalie Sinclair (1), Yanling Wang $\mathbb{D}$, Shu Xie, and Der-Ching Yang $(\mathbb{C})$
}

\subsection{Introduction}

\subsubsection{What Was Presented at the Conference: Overview}

The participants of working group 2 presented a broad range of studies, 11 papers in total, related to whole number learning representing research groups from 11 countries as follows.

Two large cross-sectional studies focused on developmental aspects of young children's number learning provide a lens for re-examining 'traditional' features of number acquisition. van den Heuvel-Panhuizen (the Netherlands) presented a coauthored paper with Elia (Cyprus; Elia and van den Heuvel-Panhuizen 2015) on a

Electronic Supplementary Material The online version of this chapter (doi:10.1007/978-3-31963555-2_7) contains supplementary material.

The first two authors wrote the chapter with the collaboration of other working group members.

J. Mulligan $(\varangle)$

Macquarie University, Sydney, NSW, Australia

e-mail: joanne.mulligan@mq.edu.au

L. Verschaffel $(\triangle)$

Katholieke Universiteit Leuven, Leuven, Belgium

e-mail: lieven.verschaffel@kuleuven.be

A. Baccaglini-Frank

Università di Pisa, Pisa, Italy

A. Coles

University of Bristol, Bristol, UK

(C) The Author(s) 2018

M.G. Bartolini Bussi, X.H. Sun (eds.), Building the Foundation: Whole

Numbers in the Primary Grades, New ICMI Study Series,

https://doi.org/10.1007/978-3-319-63555-2_7 
cross-cultural study of kindergartners' number competence focused on counting, additive and multiplicative thinking. Second, Milinković (2015) examined the development of young Serbian children's initial understanding of representations of whole numbers and counting strategies in a large study of 3- to 7-year-olds. Children's invented (formal) representations such as set representation and the number line were found to be limited in their recordings.

In a South African study focused on early counting and addition, Roberts (2015) directs attention to the role of teachers by providing a framework to support teachers' interpretation of young disadvantaged learners' representations of number when engaging with whole number additive tasks.

Some papers reflected the increasing role of neuroscientific concepts and methodologies utilised in research on WNA learning and development. Sinclair and Coles (2015) drew upon neuroscientific research to highlight the significant role of symbol-to-symbol connections and the use of fingers and touch counting exemplified by the TouchCounts iPad app.

Gould (2015) reported aspects of a large Australian large study of children in the first years of schooling aimed at improving numeracy and literacy in disadvantaged communities. A case study exemplified how numerals were identified by relying on a mental number line by using location to retrieve number names. This raised the question addressed in the neuroscientific work of Dehaene and other papers focused on individual differences in how the brain processes numbers.

The Italian PerContare ${ }^{1}$ project (Baccaglini-Frank 2015) built upon the collaboration between cognitive psychologists and mathematics educators, aimed at devel-

\footnotetext{
San Paolo of Torino.

P. Gould

NSW Department of Education and Communities, Sydney, NSW, Australia

$\mathrm{S}$. He

Hangzhou Normal University, Hangzhou, China

$\mathrm{Y}$. Ma $\bullet$ Y. Wang $\bullet$ S. Xie

Northeast Normal University, Changchun, China

J. Milinković

University of Belgrade, Belgrade, Serbia

A. Obersteiner

Technische Universität München, München, Germany

N. Roberts

University of the Witwatersrand, Johannesburg, South Africa

N. Sinclair

Simon Fraser University, Burnaby, BC, Canada

D.-C. Yang

National Chiayi University, Chiayi, Taiwan
}

${ }^{1}$ The PerContare project was coordinated by Fondazione ASPHI onlus, with the support of Compagnia di San Paolo and the operative support of Fondazione per la Scuola of Compagnia di 
oping teaching strategies for preventing and addressing early low achievement in arithmetic. It takes an innovative approach to the development of number sense that is grounded upon a kinaesthetic and visual-spatial approach to part-whole relationships.

Mulligan and Woolcott (2015) provided a discussion paper on the underlying nature of number. They presented a broader view of mathematics learning (including WNA) as linked to spatial interaction with the environment; the concept of connectivity across concepts and the development of underlying pattern and structural relationships are central to their approach.

One group of papers presented studies about other computational aspects of WNA such as the variation, efficiency and flexibility of representations and strategies for counting, mental arithmetic, written algorithms, computational estimation and word problems. Obersteiner and colleagues (Obersteiner et al. 2015) proposed a coherent five-level competence model for WNA in the lower grades of elementary school. In another study Verschaffel and colleagues (Verschaffel et al. 2015) compared two kinds of strategies for processing mental subtraction, namely, subtractionby-addition. Another three different studies provided new insights into mental and written WNA strategies and errors by students in the middle elementary grades: He (2015) focused on cognitive strategies for solving addition and subtraction problems; Yang (2015) highlighted the conceptual difficulties of students' judging the reasonableness of results in whole number calculations; Ma et al. (2015) analysed students' systematic errors for three-digit multiplication and linked these errors to teaching strategies. While these studies generated rich discussion about the range of research questions that focused on computational processes linked to WNA, these studies were not considered the main focus of this chapter, which instead articulates the ICMI23 (Theme 2) position paper.

\subsubsection{The Discussion of the Working Group}

As in most other working groups, the eight 1-hour sessions were organised in two different forms. Whereas the first five sessions were devoted to the presentation and discussion of the participants' accepted papers, the last three whole-group sessions involved discussions, wherein two major themes were discussed. First, to what extent can the currently influential neuro-cognitive perspective, as elaborated in Butterworth's plenary lecture, act as an appropriate theoretical scope to think about early mathematical development (and stimulation of that development) or whether this perspective needs to be nuanced and enriched by other perspectives? The second major point of discussion addressed the potentialities and limitations of the methodologies utilised in the studies on children's whole number learning and development being presented in the working group (and in some other working groups) and, more specifically, of (a) the design of the cross-sectional, longitudinal and intervention studies aiming at understanding how children develop competencies with whole numbers, as well as (b) the tasks that are common to many studies 
measuring the understanding of magnitude of numbers, such as number comparison and number-line estimation tasks.

Obviously, the topics dealt with in working group 2 were related to those addressed in the other working groups and panels, as will become clear through the numerous cross-references that will be provided in this chapter. However, working group 2 tried to tackle these common topics from the two perspectives mentioned above, namely, the psychological and the methodological perspectives.

\subsubsection{About the Chapter}

The chapter focuses essentially on two key aspects of the Theme 2 discussion (as presented in the Discussion Document, see the Appendix 1 to this volume) that discussed neuro-cognitive, cognitive and developmental analyses of whole number learning. Its aim is to bring these perspectives into our discussion by acknowledging the realisations and promises of neuroscientific research while adopting a critical approach from a mathematics education perspective. The structure and content of the chapter are an outcome of our synthesis of key ideas following our discussions in working group 2. Thus, the chapter will (1) present, discuss and illustrate perspectives complementary to neuro-cognitive research and (2) discuss methodologies utilised in studies on children's whole number learning and development. There are five main sections.

The bulk of the first section (Sects. 7.1.1 and 7.1.2) provides an overview of the ICMI Study 23 Conference presentations and the working group 2 discussion.

The second section (7.2) focuses on two neuro-cognitive perspectives: first, Butterworth's 'starter kit' is discussed in light of Butterworth's plenary paper (see Chap. 20) and his contribution to the working group discussions. Second, some related research on the triple-code model of Dehaene and colleagues (Dehaene et al. 2003; see also Dehaene 2011) is presented. Gould (2015) then draws upon some examples from the research of how quantities and numbers are transcoded and represented.

The third section (7.3) provides an overview of related research from cognitive perspectives that informs the discussion for working group 2. Verschaffel and Mulligan develop this overview of the literature to complement the examples provided by working group 2 participants. Cross links with examples from other themes are highlighted.

The fourth section (7.4) describes some pertinent examples of studies presented to working group 2 and applications of the perspectives described in Sect. 7.3: ordinality (Sinclair and Coles 2015), part-whole relations (Baccaglini-Frank 2015), additive relations (Roberts 2015), number competence (Elia and van den HeuvelPanhuizen 2015) and counting and representational structures (Milinković 2015).

The fifth section (7.5) discusses methodological issues common to neurocognitive, cognitive and developmental analyses of studies on children's WNA. Cross-sectional, longitudinal and intervention studies are discussed in terms 
of their appropriateness for investigating children's competence with whole number. In that section, we also discuss task design in cognitive neuroscience research pertinent to number learning.

In the sixth section (7.6), some tentative conclusions are drawn and implications for teaching and learning and further research are discussed.

\subsection{Neuro-cognitive Perspectives}

\subsubsection{A 'Starter Kit' for Early Number}

The components of Butterworth's 'starter kit' (Butterworth 2005) for early number learning are primarily focused on cardinal aspects of number and its importance for later mathematical development. In most cognitive neuroscientific studies, children's foundational competencies are related to children's general mathematical achievement as measured by standard school achievement tests of number and computation rather than other aspects of mathematical development such as spatial processes.

From a very young age, humans have an inherited core capacity for numerical processing. For example, the process of subitising refers to the immediate and accurate estimate of one to four objects without serial enumeration. Another core process is described as representing non-symbolic numerical magnitudes on a mental number line. Symbolic representations $(3,100,1 / 2,3.17 \ldots)$ are gradually mapped onto these non-symbolic representations. These magnitude representations are commonly assessed by means of subitising, magnitude comparison and number line estimation tasks. Examples are shown in Figs. 7.1 and 7.2, respectively.

Butterworth (see Chap. 20) describes these two foundational 'core systems'. Deficiencies in these core systems may contribute to low numeracy. He refers to an 'object tracking system' that has a limit of three or four objects and is thought to underlie 'subitising'. Another core system is the 'analogue number system' (ANS). 'The internal representations of different numerical magnitudes can be thought of as Gaussian distributions of activation on a 'mental number line'. It is typically tested

Fig. 7.1 Which is the larger set?

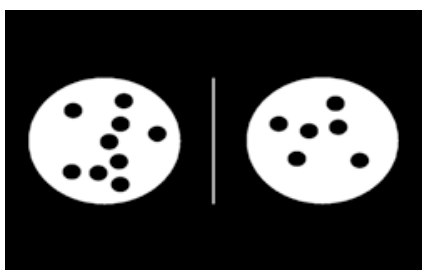

Fig. 7.2 The line begins at 0 and ends at 10 . Where is the number 6 located?

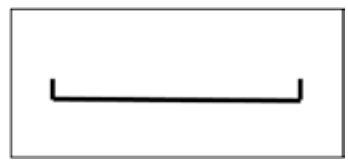


by tasks involving clouds of dots (or other objects) typically too numerous to enumerate exactly in the time available' (p. 480).

Butterworth refers to the study of Australian Aboriginal children that exploited their known visual strengths to solve accurately non-standard arithmetic tasks where they had no number words to describe the quantities (Butterworth and Reeve 2008). These children matched the spatial patterns of the addend and augend sets. The findings suggested that there are various models for number that are not necessarily one-dimensional such as in the mental number line and these can be two-dimensional in nature.

Some key findings are drawn from Butterworth's research: (1) numerical magnitude understanding is positively and predictively related to (general) mathematics achievement and (2) numerical magnitude understanding can be improved by means of game-based intervention programmes, although the transfer effects from those games to mathematics learning more broadly is still rather small (see Chap. 20).

\subsubsection{Neuropsychology and the Triple-Code Model}

Neuropsychologists have sought to understand how brain functioning influences cognition, including mathematics and whole number learning (Dehaene 2011). Simple models associated with number processing have been proposed and tested, and the main areas of the brain identified as being activated in number processing have been refined.

Dehaene et al. (2003) proposed a triple-code model of working with number, consisting of three components: verbal, visual (numerals) and magnitude. The model postulates three main representations of numbers:

A verbal code in which numbers are represented as a parsed sequence of words.

A visual Arabic code in which numbers are represented as identified strings of digits.

An analogical quantity or magnitude code.

Each part of the model has been associated with increased activity in a particular part of the brain. For example, the horizontal segment of the intraparietal sulcus has been suggested as the region involved in encoding the analogical representation of numerical magnitude (Dehaene et al. 2003).

\subsubsection{Transcoding Numerals (Symbols) to Number Words}

Dehaene et al. (2003) proposed two major coordinated routes: a direct asemantic route that transcodes written numerals, i.e. the symbolic notations, to verbal representations and an indirect semantic route for quantitative processing. Alternative 
semantic routes are those that go through an intermediate step of activation of quantity associated with the target numeral.

In the working group 2 presentation and subsequent discussion, Gould raised the question of whether transcoding numerals to number words is limited to either semantic or asemantic pathways. Gould (2015) drew upon an example of a 7-yearold child's alternative strategy for locating and naming numerals on the number line. The discussion centred on a videoed interview with a child (Electronic Supplementary Material: Gould 2017), identified as Jed, indicating that these are not the only pathways used to transcode numerals to words. Instead of using a direct asemantic route to transcode written numerals to verbal representations, Jed used a more laborious transcoding pathway to identify numerals. Jed attempted to visualise the location of numerals on an ordered line of numbers. He would then seek the corresponding number word by counting from one. His process for identifying numerals was purposeful and time intensive, but clearly not asemantic. Gould's pertinent example shows that coding pathways associated with number may be more complex than the neuro-cognitive triple-code model currently allows. Whereas previously it was thought that Arabic numerals might activate representations of magnitude automatically, other research suggests that this is not the case (Rubinsten and Henik 2005). Gould proposes that learning to identify numerals is a learnt process. For most students, transcoding Arabic numerals to words is an asemantic process, but for some, it can rely upon a process that is not instant. For example, Frederick, a 7-year-old student in his second year of formal schooling, would regularly confuse 12 and 20 . Over a period of 10 weeks, he learnt to correctly identify 12 and 20. However, to identify 12, that is, to say twelve in response to seeing it, Frederick counted from one to twelve subvocally.

Assessments of young children's counting often reveal the need to rely on the count from 1 (often subvocally) as the reliant strategy to 'reach' the required number name. These children are unable to move flexibly between different positions in the number sequence to count either forwards or backwards. When children move from producing an ordered sequence of counting words from 'one' to developing cardinal meaning, their understanding of the quantity is described as the count-tocardinal transition (Fuson 1988, p. 266). What is important here is whether these children possess either cardinal or ordinal understanding of quantity or both, e.g. '12'. The findings highlighted by Sinclair and Coles (this chapter) raise further questions about ordinality - could Jed explain how numerals are ordered in a sequence? Another important observation is that the verbal processes concerned with learning the labels for Arabic numerals appear to be critical for arithmetic development between the ages of 6 and 7 years (e.g. see Göbel et al. 2014). 


\subsection{Beyond Neuro-cognitive Approaches: Quantitative Relations, SFOR and an Awareness of Patterns and Structures}

This section provides an overview of current research that informs the research perspectives of working group 2. In this section, we will address the importance of reasoning about quantitative relations, children's spontaneous tendency to do so and their awareness of patterns and structures.

\subsubsection{Children's Early Competencies in Quantitative Relations}

It is apparent that the analysis of early mathematics-related competencies has capitalised on measures that emphasise children's numerical competencies, i.e. their subitising skills (Schleifer and Landerl 2011), counting skills (Geary et al. 1992), ability to compare numerical magnitudes (Griffin 2004) and ability to position numerical magnitudes on an empty number line (Siegler and Booth 2004). While such measures provided empirical evidence for the multicomponential nature and importance of young children's early numerical competencies (Dowker 2008), they imply a restricted view on children's early mathematical competencies and their importance for later mathematical development. Starting from Piaget's logical operations framework (e.g. Piaget and Szeminska 1952), there is a recent renewed research attention to children's quantitative reasoning skills, such as their understanding of the additive composition of number or their multiplicative reasoning skills, as well as to their importance for later mathematical learning at school (e.g. Clements and Sarama 2011; Nunes et al. 2008, 2012).

Several authors have explored the emergence and early development of these two forms of quantitative reasoning. As far as additive reasoning is concerned, various principles including the additive composition of number but also the commutativity $(a+b=b+a)$, the addition-subtraction inverse $(a+b-b=a)$ and the additionsubtraction complement principle $(a-b=. \rightarrow b+.=a)$ have been intensively studied (Baroody et al. 2009; Bryant et al. 1999; Gilmore and Bryant 2006; Robinson et al. 2006), sometimes also in relation to children's actual use of these principles in their mental arithmetic (Baroody 1999; Peters et al. 2010). However, only few studies have explicitly addressed the question of how children's understanding of these principles affects their (later) achievement in whole number arithmetic. The limited available evidence from these few studies suggests that quantitative reasoning of this sort makes a specific contribution to achievement in whole number arithmetic. These studies highlight the importance of focusing on the relational aspects of quantitative reasoning as critical principles that contribute to strong mental computational flexibility. 


\subsubsection{Spontaneous Focusing on Numbers (SFON) and Quantitative Relations (SFOR)}

The studies on the early development of children's quantitative reasoning reviewed in Sect. 7.3.1 take a typically cognitive perspective. They hardly address children's attention to, and feeling for, quantitative relations. Quantitative reasoning in these studies often relates to multiplicative thinking. Recently, researchers have started to explore children's spontaneous focusing on quantitative relations (SFOR), as a follow-up of their investigations on children's spontaneous focusing on numerosity (SFON), which has already shown to have predictive power in explaining children's later mathematical achievement (Hannula and Lehtinen 2005). McMullen, HannulaSormunen and Lehtinen (McMullen et al. 2014) describe SFOR as 'the spontaneous (i.e. undirected) focusing of attention on quantitative relations and the use of these relations in reasoning' (p. 218).

A central idea underlying these SFON and SFOR tendencies is that there are individual differences not only in how learners reason about mathematics and use their numerical skills in learning or testing situations, wherein children are guided to the mathematical elements or relations in the situation, but also how often they spontaneously focus on mathematical aspects of informal everyday situations. In these situations, the recognition and use of quantitative aspects of the situation are done at the child's own initiative and thus undirected and spontaneous (e.g. Hannula and Lehtinen 2005; McMullen et al. 2013, 2014). Therefore, studies on SFON and SFOR do not examine whether learners are able to recognise or count exact number but rather whether they spontaneously use their available number recognition or quantitative reasoning skills in situations where they are not explicitly guided or instructed to do so.

\subsubsection{An Integrated Perspective Focused on Patterns and Structures}

Mulligan and Mitchelmore (2009) looked beyond research on early numeracy and single mathematical content domains such as counting to identify and explain common underlying bases of mathematical development. Drawing on their seminal studies of multiplicative reasoning and representations of number, they investigated the cognitive development of mathematics through the assessment of children's conceptual structures. A strong body of research on patterning, early algebraic thinking and the role of spatial structuring in mathematical representations supported their integrated theoretical approach that young children could develop cognitively sophisticated mathematical concepts. Based on a series of related studies with diverse samples of 4-8-year-olds, they identified and described a new construct, awareness of mathematical pattern and structure (AMPS), that generalises across mathematical concepts and processes and can be reliably measured (Mulligan 
and Mitchelmore 2013; Mulligan et al. 2015). Interestingly, just as McMullen et al. discuss (see Sect. 7.3.2), in their conceptualisation of AMPS, Mulligan and Mitchelmore (2009, p. 39) also look beyond children's ability in developing early numerical competence, by stating that AMPS may consist of 'two interdependent components: one cognitive (knowledge of structure) and one meta-cognitive, i.e., "spontaneous" (a tendency to seek and analyze patterns)'. Both are likely to be general features of how students perceive and react to their environment, according to these authors. In line with this construct of AMPS is the discussion in working group 5 on structure, also referring to the notion of structuring for mathematical competence and the work of John Mason (see working group 5, Chap. 13).

Mathematical pattern involves any predictable regularity involving number, space or measure such as number sequences and geometrical patterns. Structure refers to the way in which the various elements are organised and related such as iterating a single 'unit of repeat' (Mulligan and Mitchelmore 2009). AMPS involves structural thinking based on recognising similarities and differences and relationships, but also a deep awareness of how relationships and structures are connected. Spatial structuring abilities provide the essential structural organisational features supporting numerical processes such as estimation of group size, multiplicative awareness of an array, iterating a unit of repeat in a repetition or equal partitions on a number line.

An interview-based assessment instrument was developed and validated, the Pattern and Structure Assessment - Early Mathematics (PASA) (Mulligan et al. 2015), across a wide range of concepts including patterning, spatial visualisation and early graphical representation. Responses included drawn representations and verbal explanations of patterns and relationships. Five broad levels of structural development were identified and described: prestructural, emergent, partial, structural and advanced structural (e.g. see Mulligan and Mitchelmore 2013). Further validation studies indicated that high levels of AMPS were correlated with high performance on standardised achievement tests in mathematics with young students (Mulligan et al. 2015). The PASA yields an overall AMPS score as well as scores on five individual structures (sequences, shape and alignment, equal spacing, structured counting and partitioning). All of these structures are highly interrelated. Repeating pattern sequences, equal spacing and structured counting all involve the idea of equal groups or units; shape and alignment considerations often result in equal groups; and partitioning requires the construction of equal groups or parts. In Chap. 16, a description of these structural groupings is provided in view of identifying common characteristics of AMPS that are often lacking in children with mathematics learning difficulties (MLD).

In alignment with the assessment of AMPS, an innovative, highly challenging alternative learning programme, the Pattern and Structure Mathematics Awareness Program (PASMAP), was developed and evaluated longitudinally in the kindergarten (the first year of formal schooling in Australia). This study provided the empirical evidence that young children are capable of representing, symbolising and generalising mathematical patterns and relationships, albeit at an emergent level (Mulligan et al. 2013). These findings suggest that restricting early learning to basic 
counting, simple arithmetic and informal notions of measure and geometry limits the development of AMPS. The study also tracked and described children's individual profiles of mathematical development, and these analyses showed that core, underlying mathematical concepts are based on AMPS and that some students develop these more readily and in more complex ways than others.

The PASMAP programme develops integrated learning experiences aimed at promoting visual memory, abstraction and generalisation, suitable for young students. Each PASMAP learning 'pathway' is directed mainly towards one or more of the five core structural groupings described above. The initial pathways include pattern as unit of repeat and growing patterns, grid structure, two-dimensional and three-dimensional relationships, structuring base ten, partitioning and sharing, equal grouping, unitising in measurement and symmetry and transformations. The first pathways are followed by more challenging tasks that link with the previous pathways and extend to multiplicative patterns, metric measurement, patterns in data and angles, direction and perspective taking. Clearly there is a strong thread of spatial structuring inherent in the pathways of learning.

In summary, there are strong connections among the theoretical approaches discussed in this section. They all highlight the importance of quantitative relations, patterns and structures as fundamental to whole number arithmetic. Recognising children's spontaneous attention to quantities can be linked to the development of AMPS that also focuses on children's natural tendency to seek structure in forming numerical relationships. In this respect, it is interesting to point to the paper presented by Sayers and Andrews (2015), which was presented in working group 1, but which also addressed the question of the foundations of number sense in a remarkably broad way. By summarising the recent research work in this domain, these authors arrived at a multidimensional framework, which they have called foundational number sense (FoNS), that comprises the following eight categories: number recognition, systematic counting, awareness of the relationship between number and quantity, quantity discrimination, an understanding of different representations of number, estimation, simple arithmetic competence and awareness of number patterns. This framework provides us, to some extent, with a comprehensive picture of early number competence that was also discussed in working group 2 . The features that are not evident in this framework, i.e. awareness of mathematical patterns and structure (AMPS) and spontaneous focusing on number (SFON) and on relations (SFOR), are taken up in the working group 2 discussion.

\subsection{Exemplars of Classroom Studies from Cognitive Perspectives}

In this section, we describe some pertinent examples of intervention studies presented to working group 2 or other applications of the perspectives described in Sect. 7.3: ordinality (Sinclair and Coles 2015), the Italian PerContare project 
focused on structural relationships in arithmetic (Baccaglini-Frank 2015), improving early numeracy through additive relations (Roberts 2015), a cross-cultural study of kindergartners' number competence (Elia and van den Heuvel-Panhuizen 2015) and counting and representational structures (Milinković 2015).

\subsubsection{Ordinal Awareness in Learning Number}

For studies on ordinality, we draw on the work of Sinclair and Coles (2015). This raises an important question concerning typical developmental sequences posited by theories of early number learning, where what is given emphasis in the first years of schooling is training children to associate numbers with counting and matching sets of objects. Their research has led to the hypothesis that what is significant in the learning of number (and mathematics more generally) is not being able to link symbols to objects in a manner that is often considered accessible or natural but being able to link symbols to other symbols.

Sinclair and Coles (2015) make a distinction, in relation to number, between ordinal and cardinal aspects. They refer to ordinality as the capacity to place number words and numerals in sequence: for example, to know that 4 comes before 5 and after 3 in the sequence of natural numbers. Other aspects of ordinality, such as the use of ordinal names and symbols such as 'first' and '1st', may be common, for instance in the French tradition. Cardinality refers to the capacity to link number symbols to collections, e.g. to know that ' 4 ' is the correct representation to denote a group of four objects. They assert that the current emphasis on cardinal awareness in learning number may be misplaced (Coles 2014) and they have been exploring what is involved in developing greater ordinal awareness of number and what are the potential benefits?

Recent neuroscientific studies (e.g. Lyons and Beilock 2011) have challenged the dominant cardinal view of numerical cognition. Lyons and Beilock found a 'distance effect' persisted with the order comparison of groups of dots, but, importantly, when judging the order of numerals, the distance effect is reversed. In other words, when asked if three numerals are in order, the closer they are together, the quicker it is found that subjects can typically make the judgement of correct ordering or not. Lyons and Beilock used this reversal of the distance effect to suggest that the brain is doing something different when making ordinal comparisons of numerals, compared with both cardinal comparisons (of numerals or dots) and compared with ordinal comparisons of dots.

A common approach to working on ordinality in schools involves practising the number song; children are invited to count in ones up to 5 or 10, then 20 and then 100. While Sinclair and Coles see much value in this practice, as a first way of introducing children to the language and sounds of numbers, working on the successor function for integers does not exhaust the potential of ordinal awareness. This has already been made evident in the work of Gattegno (1974), whose curriculum for early number was based on developing awareness of relations among lengths, where 
what is symbolised are relations between objects (greater than, less than, double, half), rather than, say, using numerals to label 'how many' objects are in a collection. Gattegno introduced work on place value as a linguistic 'know-how' and not something that required 'understanding'. Similarly, the research discussed in working group 1 (Chap. 5) and also in Chap. 3 refers to the important role of language in labelling numbers. Gattegno also made extensive use of fingers (both the teacher's and the children's) as haptic symbolic devices for working on number relations, with a focus on correspondence and complementarity. Sinclair and Coles see awareness of number, in this curriculum, arising out of linguistic skill and awareness of relations in a manner that does not emphasise nor require a cardinal focus on counting collections.

Sinclair and Coles direct attention to the importance of ordinality in researching the use, in the context of the early learning of number, of an innovative iPad app, TouchCounts (Sinclair and Jackiw 2011). The discussion in Chap. 9 also refers to the use of TouchCounts as a tool for learning. TouchCounts was initially designed as a counting environment, to help children learn about one-to-one correspondence. Every time a finger touches the screen, a yellow disc appears, labelled with a numeral, and that numeral is spoken aloud. Each subsequent touch produces a yellow disc with the next numeral on it. With the gravity mode turned on, taps that are made by the child below the 'shelf' fall away, much in the same way that turning the page of a book makes that page number disappear. If one taps above the shelf, the yellow disc is 'caught' and remains on the shelf. It is thus possible to see just the yellow disc labelled ' 6 ' on the shelf if the previous five taps have been below the shelf. Notice that this task requires being aware of the fact that 5 comes before 6 , but does not require any sense of cardinality. In both the temporal dimensions, but also because of the lack of cardinal reference, this Enumerating World emphasises ordinality. With the use of the aural feedback, as well as the numerals, there is also a strong emphasis on language and symbol, as per Lyons' recommendation (see Sinclair and Pimm 2015).

We draw upon this example of Sinclair and Coles from TouchCounts that points to the potential for ordinality in learning number (this volume, Sect. 9.3.5.3). In a kindergarten classroom, the children are sitting on the carpet, with the overhead projector hooked up to TouchCounts. The teacher has asked the children to count by $5 \mathrm{~s}$. They do this by tapping with four fingers (simultaneously) below the shelf and then once above. This leaves the multiples of 5 on the shelf. The children take turns doing the $4+1$ tapping, but were asked to announce the number that would be on the shelf before starting tapping. Note that instead of hearing 'five, ten, fifteen...', the children hear 'four, five, nine, ten, fourteen, fifteen...'

The teacher had intended to only get up to about 25 , but the children wanted to keep going. At 125, they began to predict what number would appear on the shelf chanting it out, chorus style - and ended up going all the way to 200. At this point, the following interaction took place:

Cam: I thought that two hundred was right after one hundred, but it's not.

Teacher: No, how far is it away from one hundred?

Cam: It's, it's, it's one more hundred away. 
Significant in this episode is the fact that the children were involved in a skipcounting activity that had no explicit connection to a quantity of objects. Instead of seeing five objects as a cardinal quantity, they only saw the numbered object 5, as with all the multiples of 5. The attention was focused on the structure of the numbers, which is what enabled the children to begin to chant out the multiples. When the class reached 200, there had been no connection made between the number word and a quantity (of, say, two hundred objects). Indeed, Cam's realisation about the relation between 200 and 100 is not a cardinal one; he is instead basing his understanding of the relation on the observation that all the same multiples of 5 have to be done again in order to get from 100 to 200. In this sense, the relation seems to be deeply temporal, assembled as it is with the time it takes to create all the numbers up to 100 and then to 200. The relation is also entangled with TouchCounts's pronouncements ('one hundred', 'one hundred and forty-seven', 'two hundred'), some of which these children would never had heard before and which they could not have read from the symbolic forms $(100,147,200)$, but could now begin to associate with those forms.

The discussion above suggests it is important to balance ordinal and cardinal aspects of number sense development in the primary grades. This will require some reflection on the ingrained ways in which cardinality is now privileged, as well as further creative explorations of how ordinality can be mobilised to promote the development of other number-related awareness such as place value.

\subsubsection{Part-Whole Relations and Structure Sense}

The Italian project (Baccaglini-Frank and Scorza 2013; Baccaglini-Frank and Bartolini Bussi 2015) builds upon a collaboration between cognitive psychologists and mathematics educators, aimed at developing teaching strategies for preventing and addressing early low achievement in arithmetic (also see papers by YoungLoveridge and Bicknell 2015, and Gervasoni and Parish (2015). This project takes an innovative approach to the development of number sense, that is, being grounded on a kinaesthetic and visual-spatial approach to part-whole relationships.

The project focuses on the importance of perceiving part-whole relationships and of becoming aware of structure (Baccaglini-Frank 2015; Electronic Supplementary Material: Baccaglini-Frank, 2017a). This demonstrates that partwhole relations arise from what Resnick and colleagues (Resnick et al. 1991) have described as proto-quantitative part-whole schemas 'that organize children's knowledge about the ways in which material around them comes apart and goes together' (p. 32). For example, part-whole thinking helps students recognise that numbers are abstract units that can be partitioned and then recombined in different ways to facilitate numerical calculation (Britt and Irwin 2011). Moreover, part-whole thinking is fundamental for higher mathematical reasoning. For example, the pre-algebra literature highlights how if attention is drawn to the development of part-whole relations, no longer do 'addition and subtraction appear as separate operations, but 
rather as dialectically interrelated actions that arise from the part-whole relation between quantities' (Schmittau 2011, p. 77).

Baccaglini-Frank (2015) refers to the part-whole relationship as a construct, highly resonant with the research on awareness of mathematical pattern and structure (AMPS) (Mulligan and Mitchelmore 2009, 2013). She refers to the critical feature of AMPS that is characterised by the child's structuring of groups to represent quantities; this involves part-whole relationships. Similarly, the ability to structure quantities is discussed in working group 5 (see Chap. 13) where the focus is on instruction that needs to provide opportunities for structural relationships to be associated with fundamental properties.

The following examples illustrate the use of hands and fingers to represent structure and the use of partitioning in the context of multiplication.

\subsubsection{Hands and Fingers: An Important Embodied Structure}

Various studies have highlighted how sensorimotor, perceptive and kinaesthetictactile experiences are fundamental for the formation of mathematical concepts even highly abstract ones. For example, the key role attributed to the use of fingers in the development of number sense seems to be highly resonant with the frame of embodied cognition. Fingers and hands naturally embody part-whole relationships with respect to 5 and 10 and therefore can and should be used to foster such awareness. The didactical potential of hands and fingers in their natural positions can be exploited in many different ways (e.g. see Baccaglini-Frank 2015), including through multitouch technology, well before formal schooling starts (e.g. BaccagliniFrank and Maracci 2015).

\subsubsection{Use of Artefacts for Fostering the Development of Structure Sense: The Importance of Sharing Strategies}

Various studies in mathematics education have focused on the design and implementation of didactical activities significantly based on bodily experience and on the manipulation of concrete objects with the aim of fostering the development of particular mathematical meanings. For example, in Chap. 9 (Sect. 9.2.1), within a semiotic perspective, Bartolini Bussi and colleagues describe how the student's use of specific artefacts in solving mathematical problems contributes to his/her development of mathematical meanings, in a potentially 'coherent' way with respect to the mathematical meanings aimed at in the teaching activity (Bartolini Bussi and Mariotti 2008). However, they argue that it is important to keep in mind that in fostering the development of mathematical meanings an essential component is the students' sharing, comparing and evolving of strategies (which can be accomplished in a number of different ways). These mathematical meanings, of course, can include structure sense, and this can be promoted through a variety of different mathematical content. 
Fig. 7.3 A possible decomposition of $7 \times 3$ into $5 \times 3+2 \times 3$

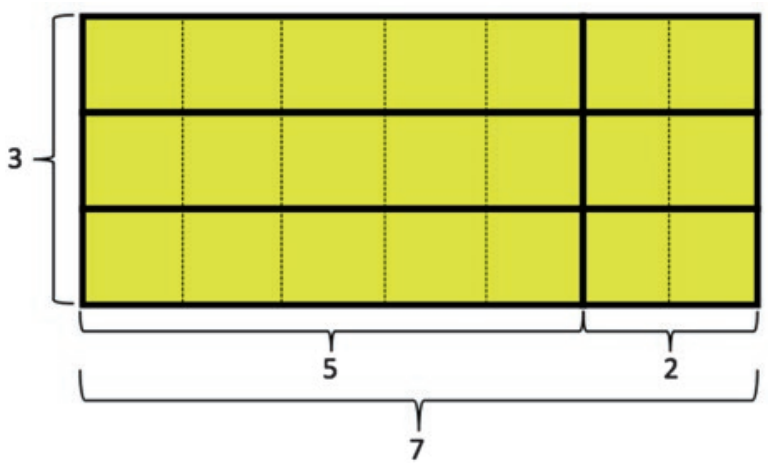

As an example, Baccaglini-Frank (2015) shows how 7-year-old students learned to think about (and perform) products (up to $10 \times 10$ ) within the Italian PerContare project. The children were introduced to rectangle diagrams, cardboard rectangles with a grid of $1 \mathrm{~cm}^{2}$ squares marking its dimensions, which represent the numbers to be multiplied. The area of the rectangle (and its unit of measure) is the number of squares that make it up. Various activities with the rectangles are proposed with the didactical goal of fostering the students' production of visual and kinaesthetic-tactile manipulative strategies for calculating products using number facts they already know. Typically, students in the experimental second grade classes already knew the sequences of the first 10 multiples of 1 (from counting), 2 (they had learned to 'double'), 5 (they could quickly add 'hands') and 10 (they could quickly count up bundles of straws). So the activities aimed at developing strategies of decomposition and composition based on such knowledge. For example, to calculate $7 \times 3$, children could think of 7 as $5+2$ and use the known rectangles $5 \times 3$ and $2 \times 3$ to build the total rectangle. Figure 7.3 shows an example.

The different strategies used by the children were compared and discussed. By the end of the school year, many children were able to perform calculations without the support of the physical rectangle diagrams any longer. For example, below is the verbal description produced by Marco ( 7 years 8 months) of the mental (and highly visual) strategy he uses to figure out $7 \times 8$ when he is called on by the teacher.

Teacher: Without drawing the 'building ${ }^{2}$ seven times eight, can you tell me how you break it and count it?

Marco: So, seven times eight... I break it into five and two, and I count it: five, ten, fifteen, twenty, twenty-five, thirty, thirty-five, forty... and I already have forty. Then I count the twos: two, four, six, eight, ten, twelve, fourteen, sixteen. Then I do forty plus, uh, I break the sixteen into ten and six, and I do forty plus ten, fifty, then I add those six and it is fifty-six.

Teacher: Wow! You are tremendous!

\footnotetext{
${ }^{2}$ In this class the teacher took on the class' idea to refer to the rectangle diagrams as (apartment) 'buildings' which could be split and put back together.
} 
As he speaks, Marco frequently gazes into space, as if he were seeing the diagram he is decomposing and recomposing. Mulligan et al. (2013) refer to this as visualising the structure, a central component of AMPS.

The examples provided by Baccaglini-Frank (2015) turn our attention to the critical role of the structure of artefacts and the ways that young students interpret and construct representations. In the case of Marco, he has internalised the visualised 'structure' of the diagram, and we can infer that he had internalised the structure of the grid. The use of structure sense is embedded within this example. The ability to decompose or partition mathematical representations is directly linked to the child's strategies for calculating, often articulated by the child's strong visual imagery of buildings to be broken up and through verbalisation of 'I break... into parts'. The key process here is not counting by ones or repeated addition but structuring by partitioning or 'breaking up'. Some knowledge of base-ten structure is also evident here. Here we see similarities with the work of Young-Loveridge and Bicknell in their paper discussing the role of structure in terms of place value and grouping (working group 3, Chap. 9, this volume).

\subsubsection{Additive Relations}

The study by Roberts (2015) complements the work of Baccaglini-Frank (2015) pertaining to part-whole and structure sense. Roberts presented to working group 2 a conceptual framework for interpreting children's external representations of whole number additive relations in the early grades (Roberts 2015). She bases her approach on growing evidence from classroom studies in South Africa that one of the major factors inhibiting learners' mathematical progression is continued using of counting by ones strategies for mathematical calculations. This concern is not exclusive to studies with South African teachers and young children; for example, YoungLoveridge highlights the same concern in her studies from New Zealand reported in Chap. 13 (this volume).

Roberts explores young learners' representations of additive relationships and provides insights into underlying structure linked to grouping. She presents an adapted framework focusing on shifts within modes of representation that denote a move from counting to calculating. Based on the work of Ensor et al. (2009), she presents the adapted framework moving from concrete apparatus to iconic images, to indexical images (generic), to symbolic (number-based) and to the abstract symbolic-syntactical level. What the study suggests is that progression within these dimensions varies when various modes of representation are used for different tasks and at different times over the 10-day intervention period. What Roberts proposes is that it is important for teachers to attend to both structure (arrangement and groupwise) and action within a particular mode of representation, when interpreting learners' representations of additive relations.

Roberts' approach that articulates the need for children to work flexibly with multiple modes of representation is exemplified; particular representational types 
are not automatically mapped to a particular calculation strategy. How the interplay of the various modes of representation interrelates with the developing sense of structure and the complexity of the structural features of the tasks at hand is exemplified. However, what we see here is the attempt to integrate complex aspects of structural development with more traditional, broad levels of progression from concrete to abstract thinking, as well as consideration of embodied action. What is difficult to assess is whether the structure dimensions direct or dominate the influence of other dimensions. There is clearly emphasis on the use of colinearity (left-right, top-bottom), linear directions and partitioning. This complex matrix approach raises relevant research questions about how the internalisation of the structural features occurs within and across the four dimensions over time and how this promotes abstraction and generalisation in developing arithmetic relationships such as equivalence or commutativity.

\subsubsection{Cross-Cultural Study of Number Competence}

Elia and van den Heuvel-Panhuizen investigated the number competence of kindergartners from the Netherlands $(n=334)$ and Cyprus $(n=304)$. The study supported the multidimensional nature of kindergartners' number development. Although the study did not include assessment items from the full domain of number and operations, four structures were found to be central to number competence: counting, subitising, additive and multiplicative reasoning. The children from the Netherlands outperformed those from Cyprus, demonstrating competence across the four components. The number competence of the children from Cyprus reflected two components, including extended counting and additive reasoning. The discussion focused on possible reasons for differences in competence where it was considered that the Cyprus kindergarten's mathematics curriculum and teaching practices may have been restricted to counting and additive reasoning and less attention was placed on subitising and multiplicative reasoning. Counting strategies may have dominated the Cyprus children's strategies. It was clear that young kindergarten children could solve multiplicative items and they connected multiplication and divisions processes, although the multiplicative items were the most difficult. This was consistent with other studies presented to working group 2.

Mulligan and colleagues reported similar findings to those of the Netherlands sample in their assessment of counting, subitising and multiplicative reasoning with kindergarten students from Australia using the interview-based Pattern and Structure Assessment (PASA). The possible over-reliance on counting was also described in the studies by Roberts and Milinković. Here we refer to the study of Gervasoni and Parish (2015), where they used individual interviews to assess over 2000 Australian primary-aged children from grades $1-4$. Counting, place value, additive and multiplicative tasks were administered with gradual increase in competence, but an overreliance on counting strategies even at grade 4 level was found. The working group 2 also questioned the limitations of some early number competency assessments 
that may restrict items to counting and additive processes and argued for more assessments to probe mental calculation strategies.

\subsubsection{Counting and Representations of Number}

Milinković examined the development of young Serbian children's initial understanding of representations of whole numbers and counting strategies in a large cross-sectional study of 661 children aged 3-7 years. Individual interviews were conducted with a consistent set of 24 tasks across the sample so that developmental patterns in performance based on age categories could be ascertained. Although many of the tasks replicated a traditional approach, such as focusing on counting, set representation and one-to-one correspondence, there were some tasks that focused on structure through different spatial arrangements of groups of objects. Further there were tasks that required children to complete a two-dimensional drawing to show a quantity (box task) and to extend numbers on a number line that did not include equidistant points to assist in this process.

Although the research report is limited to performance data across the sample, there are some critical features inherent in some tasks that relate to other research discussed in working group 2. Milinković highlights the analysis of children's understanding of different graphical representations - the box diagram and number line - and presents some pertinent examples. Representations such as sets and the number line were found to be limited in their recordings. The ability to use equal spacing or a composite unit (equal size) to represent number appeared most difficult in the sequence of tasks developmentally.

\subsection{Methodological Issues and Recommendations}

In this section, we examine methodologies utilised in the type of studies on children's whole number learning and development being reviewed in this section. Evidently, these methodologies do not cover the whole range of research methods being used in the domain of WNA. Rather than providing a broad overview of the topic, we focus on two issues. First, we discuss study designs and their potentialities and limitations for understanding how children develop competencies with whole numbers. The discussion is restricted to cross-sectional, longitudinal and intervention studies. Second, we discuss task designs in cognitive neuroscience research pertinent to number learning. Here, we focus on the validity of tasks that are common to many studies measuring the understanding of magnitude of numbers. Such discussion reflects some of the issues raised in the previous ICMI Study 22 in 2014 (Watson and Ohtani 2015). To illustrate methodological issues, we refer to key aspects of whole number learning, such as strategy use, developmental aspects and 
the effectiveness of instructional approaches. We conclude with some recommendations for further research on whole number learning.

\subsubsection{Study Designs}

\subsubsection{Assessing Strategy Use with Cross-Sectional Studies}

Whole number learning requires, among other things, learning increasingly advanced strategies. For example, young children might initially use counting strategies to solve addition tasks, but they might later use stepwise addition strategies or they might be able to retrieve the results from memory by retrieval of known number facts. Cross-sectional studies allow insights into children's performance and strategy use at a particular point in time. They also allow for investigation of how performance and strategy use depend on specific types of tasks and how performance and strategy use vary between students with different educational and sociocultural backgrounds (e.g. He 2015; Ma et al. 2015; Milinković 2015; Verschaffel et al. 2015; Yang 2015). Chapter 3 on language aspects, Chap. 5 on reporting the discussion of working group 1 and the commentary paper by David Pimm (Chap. 4) each address the role of language and culture, also from a historical perspective in the development of whole number arithmetic. Here, we turn attention to the working group 1 discussion that shows how differences in number names according to culture may lead to wide differences in learning and pedagogical strategies.

An important issue in studying strategy use is the interplay between individual strategy use, individual ability and the affordances of a specific task. For example, children might not always use the most sophisticated strategy they could possibly use if less sophisticated strategies are more efficient for the specific task at hand. On the other hand, students might not be able to adapt their known strategy to the specific task. For that reason, researchers have argued that if in the assessment situation students are allowed to select their preferred strategy, we cannot draw valid conclusions concerning strategy efficiency (Siegler and Lemaire 1997). To draw such conclusions, it is necessary to compare students' performance in a choice condition wherein they are free to select their preferred strategy to their performance in a nochoice condition wherein students are forced to use a particular strategy. Many researchers have used this choice/no-choice method to study strategy efficiency and strategy flexibility (e.g. Verschaffel et al. 2015). This line of research has produced interesting and sometimes surprising results. For example, it seems that students do not always use the most efficient strategies that they may have acquired through instruction at school. Students also rely on strategies they have not been taught, and they might even invent their own strategies.

There is a rich and diverse range of studies that have examined strategy development and strategy use in early arithmetic development (e.g. see the papers by He on Chinese students' cognitive strategies to addition/subtraction problems (He 2015) and Yang on students' ability to judge the reasonableness of computational strate- 
gies (Yang 2015)). Many studies on strategy use have also focused particularly on promoting numeracy programmes or frameworks. However, to date, the complex interplay between the factors that influence strategy use on WNA tasks is not fully understood.

An important limitation of cross-sectional studies is that they do not allow conclusions to be drawn with respect to individual development or causal relations to be determined between foundational or natural abilities and mathematical learning. For that purpose, we need longitudinal studies, intervention studies and those that track, possibly from the origins, the growth of individuals' strategy development in WNA.

\subsubsection{Tracing Individual Development with Longitudinal Studies}

Longitudinal studies rely on data from individual children assessed over a longer period of time. In the case of numerical development, such studies allow for identifying those variables assessed early in development that are most predictive of later arithmetic achievement. While longitudinal studies on arithmetic development have been relatively scarce until two decades ago, an increasing number of longitudinal studies have been carried out since (e.g. see the above-mentioned synthesis of research on early number sense by Sayers and Andrews 2015). Many of these studies have produced converging results. For example, several studies found that at pre-school age, counting and linking quantity to number words are important predictors of mathematical achievement in the first years of primary school (Aunio and Niemivirta 2010; Krajewski and Schneider 2009). Other researchers have combined several measures basic understanding of numbers with the concept of number sense. Number sense, measured at the beginning of schooling, predicted achievement in school mathematics in the first and third grades (Jordan et al. 2010). In a 6-year longitudinal study, Reeve, Reynolds, Humberstone and Butterworth (2012) clustered children at the age of 6 years according to their basic numerical abilities such as dot enumeration and number comparison. The authors found that the clusters were relatively stable over the period of the study and that membership to a cluster was a robust predictor of arithmetic ability 5 years later.

Although longitudinal studies have contributed to our understanding of how certain arithmetic abilities develop over time, most of these studies have focused strongly on cognitive variables related to mathematics while paying less attention to general cognitive variables (such as IQ and working memory) or environmental variables (such as school environment, classroom teaching or socio-economic variables) (but see Skwarchuk et al. 2014). From a mathematics education point of view, this is problematic, because these more distal variables might strongly influence children's development.

The benefit for mathematics education of identifying the most relevant early predictors of arithmetic competencies is that we can develop teaching approaches that specifically address these predictors. Yet, we need further research to evaluate whether the developed teaching approaches are actually effective and to identify the 
most effective one(s) among competing teaching approaches. Intervention studies are suitable for that purpose.

\subsubsection{Evaluating Teaching Approaches with Intervention Studies}

Intervention studies have the advantage that, if properly designed, they allow conclusions about the causal effects of specific factors. This is at least the case when the intervention conditions are highly similar with respect to non-relevant factors. When designing an intervention study, an important question is how to design the control group condition. The challenge is that the question of what we want the experimental condition to be compared with to is not always obvious. Consider a game-based intervention study in which the experimental group uses a computer game that includes carefully designed number tasks. As a control condition, one might want to vary the method of instruction (computer-based versus not computerbased), the specific tasks (innovative tasks versus traditional tasks), the entertaining nature of instruction (game versus no game), the instructional setting (collaboration versus individual) or other factors. However, it is often impossible to vary all these factors within the same study. In addition, there might be theoretical reasons why combining certain factors is not reasonable from a mathematics education point of view. For example, collaborative learning might be more reasonable when the students work on problem-solving tasks than when they try to memorise arithmetic facts. Moreover, instructional factors are often closely related to one another, so that manipulating one factor can affect another factor.

Although strictly controlling the intervention conditions is necessary to draw conclusions about the causal effects of specific factors, doing so might reduce the external or ecological validity of the study. The reason is that the effectiveness of teaching approaches under controlled conditions might not transfer to regular, much more complex learning situations. Ideally, we need both highly controlled intervention studies and less strictly controlled classroom evaluation studies in order to compensate for the disadvantages of each. This will require replicating studies in a variety of settings and combining a variety of research methods (Schoenfeld 2007; Stokes 1997).

\subsubsection{Task Designs}

As discussed earlier in the first section of this chapter, neuroscience studies have addressed the brain mechanisms that underlie number processing. A main conclusion from this research field is that the human brain seems well prepared for processing (numerical) magnitudes. Although understanding number magnitudes has been a matter of research long before neuroscience studies identified the relevant brain areas, this conclusion increased the attention researchers paid to processing numerical magnitudes. Likewise, although the relation between mathematical 
abilities and other cognitive abilities has been studied for a long time, the fact that the intraparietal sulcus is a brain region responsible for magnitude processing as well as spatial thinking has influenced a number of studies addressing the relationship between numerical and spatial abilities (e.g. Mulligan and Woolcott 2015).

Although there is no doubt that understanding numerical magnitudes is an important facet of whole number arithmetic abilities, its particular role for arithmetic development is not completely clarified yet. One reason among others is that the tasks (measures) that have been used in previous studies for assessing magnitude understanding may not be as valid as many have thought them to be. Most studies have used number comparison tasks or number line estimation tasks to assess magnitude understanding. In number comparison tasks, one has to decide which of two numbers is numerically larger. In number line estimation tasks, one has to place a given number in the correct position on an empty number line. Performance on both the number comparison task and the number line estimation task has proven to be highly predictive of mathematical learning (e.g. Booth and Siegler 2008). Many researchers have concluded that processing numerical magnitudes is essential for learning of numbers and they have used either task to assess magnitude understanding. Surprisingly, studies that addressed the relation between different measures challenged the assumption that these different measures rely on the same cognitive mechanisms. Studies documented that the correlation between performance on number comparison tasks and number line estimation tasks was very small (Sasanguie and Reynvoet 2013) and that performance on symbolic and non-symbolic number comparison tasks was virtually unrelated (Gilmore et al. 2011). Meanwhile, there is converging evidence that the association between number comparison and arithmetic competence is much stronger for symbolic than non-symbolic measures (Sasanguie et al. 2014; Schneider et al. 2017), suggesting that it is the proficient use of number symbols that has a strong association with arithmetic competence.

Recent studies question the assumption that number comparison and number line estimation are 'pure' measures of magnitude understanding. A possible explanation could be that depending on the specific numbers involved, these tasks can be solved by strategies that vary in how strongly they require magnitude understanding. For example, for comparing two-digit numbers, one can rely on digit-by-digit comparison without taking into account the magnitudes of the numbers as a whole. Likewise, researchers have argued that non-symbolic number comparison tasks may not only measure magnitude understanding as such, but also the capacity to suppress irrelevant visual cues (Clayton and Gilmore 2015) or to switch the focus on reliable cues (Gebuis and Reynvoet 2012). For number line estimation tasks, the strategies one can use also depend strongly on the specific numbers. Finding the correct position of 50 on a number line from 0 to 100 is easy (because finding the midpoint of the line is a visually simple task), while finding the correct position of 83 is more difficult, because there is no clear benchmark that can be used. Recent research has documented that already second grade children use a variety of strategies for solving number line estimation tasks and that these strategies depend on modes of presentations and the availability of benchmarks (Peeters et al. 2015). 
More generally, most cognitive and neuroscience studies have used very simple tasks. For example, studies on the neural underpinnings of mental arithmetic have often used single-digit addition tasks. Although these are important first steps, and although performance of these tasks might be related to mathematical achievement later on, studying these tasks is not sufficient to explain mathematical thinking, which is typically much more complex. Accordingly, we should be cautious about interpreting neuroscience findings in terms of educational implications. In particular, we should not consider neuroscientific data as more convincing or informative than behavioural data (Beck 2010; De Smedt et al. 2011).

\subsubsection{Conclusions: Methodological Issues}

Some tentative conclusions and implications can be gleaned from the discussion provided in the above section. Studies that aim to provide predictive factors for math-related competencies may need to take into account the influence of a broader range of variables such as IQ, working memory and socio-contextual factors. The analyses of specific tasks such as number line estimation do not reflect the wide variation in children's own strategies that they may impose on the tasks. For example, the type and size of numbers may vary, but solutions may also depend on modes of presentation and the availability of benchmarks. Most cognitive and neuroscience studies have used very simple tasks limited to one area of competence. The limitations of these findings should be acknowledged in view of the much more complex relationships between concepts and processes that contribute to mathematics learning and thinking. Further, we need longitudinal studies to understand better how numerical abilities develop over time.

\subsection{General Conclusions and Implications}

\subsubsection{General Conclusions}

This chapter has highlighted the need to review neuro-cognitive, cognitive and developmental approaches to number learning and the measurement of numerical abilities. While critical components of WNA may differ between disciplines, some important commonalities have been found between approaches. Butterworth's research (2015) focused on the 'starter kit' for number also reflected conceptual foci on studies on early number from a cognitive mathematics education perspective. Tasks that incorporate subitising and numerical estimation are common to mathematics education psychological studies but differ methodologically. Although these neuro-cognitive studies provided convincing evidence of specific features of early 
number development, these were limited to numerical magnitude and cardinality, assessed in clinical studies. Cognitive studies on early numerical and general mathematical competencies have received inadequate attention in the neuroscience field.

From various cognitive perspectives, key components and processes integral to mathematics learning and related to WNA were described: spatial reasoning and spatial sense, reasoning about quantities and relationships, SFON and SFOR, structural relations and patterns (AMPS), ordinality, partitioning and representing numerical relationships. These studies, together, provide evidence that young children are capable of quantitative reasoning from a young age. In particular, Sect. 7.3 highlighted recent research on young children's capacity to reason about quantitative relations (SFOR), as well as their spontaneous tendency to do so. This line of research shows strong synergies with the structural approach to early number development that focuses on awareness of mathematical patterns and structures (AMPS). In their conceptualisation of AMPS, Mulligan and Mitchelmore also go beyond the idea of early numerical competence based on ability. AMPS consists of two interdependent components: one cognitive (knowledge of structure) and one metacognitive (a tendency to seek and analyse patterns). It seems that reasoning about quantities and relationships, SFON and SFOR, structural relations and patterns (AMPS), ordinality, partitioning and representing numerical relationships are related to structural development in mathematics.

The exemplars of studies presented in Sect. 7.4 each reflect the need to take a more integrated approach to early WNA development. The studies point to a common approach that seeks to reveal the deep interconnected structural features of conceptual development of number. The studies of Baccaglini-Frank and colleagues turn attention to the critical role of the structure of artefacts and the ways that young students interpret and construct representations. The use of structure sense is embedded within most examples. The ability to decompose or partition mathematical representations is also featured in the work of Milinković and colleagues. Several studies reflected that importance of varying models of representation supporting the idea of complexity of the learning process that is often absent from neat theoretical frameworks of likely learning progression. The discussion of methodological issues in Sect. 7.5 raises questions for future research and practice.

There were several papers in this working group which focused on learners with special needs: Butterworth (2015) drew attention to the prevalence and diagnosis of dyscalculia; Baccaglini-Frank's paper (2015) reported on an intervention study in Italy designed to redirect Italian learners at risk of dyscalculia diagnosis; Gould's study (2015) focused on one child who used an atypical way of counting; and the Roberts (2015) paper was motivated by the prevalence of South African children aged 10-12 years using such inefficient unit counting strategies (long after this was developmentally appropriate). These papers depicted groups and individual children not progressing mathematically as expected in relation to their peers and/or the national mathematics curriculum. Issues concerning children with special learning needs are discussed further in Chap. 16. 


\subsubsection{Implications for Further Research and Practice}

The chapter has highlighted three issues that lead to recommendations for further research. Firstly, we need longitudinal studies to better understand how numerical abilities develop over time. These studies should take into account not only mathrelated variables but also other variables that have crucial effects on development, such as IQ, working memory and contextual variables such as socio-economic factors and school environments. This would be helpful in putting the relevance of specific, math-related predictors into perspective. Although cross-sectional studies can hint at relevant relationships between specific sub-competencies, only longitudinal studies support conclusions about children's individual development and the causal relations between foundational math-related abilities and whole number arithmetic learnt at school.

Second, there is a need for intervention studies to develop evidence-based instructional tasks, tools and techniques. This would enhance educational practice and also contribute to our better understanding of the causal effects of arithmetic development. Combining both strictly controlled experimental studies and less strictly controlled field studies allows us to overcome the drawbacks of each (Schoenfeld 2007).

Third, we need more studies that systematically evaluate the validity of simple number tasks that have been used frequently in (neuro-)cognitive studies. A multimethod approach (as used in the study by Peeters et al. 2015) seems promising for that purpose. Once appropriate tasks and methods are available, future neuroscience studies could address more complex mathematical thinking.

A less (neuro-)psychologically dominated and more interdisciplinary approach might bring a broader, more balanced perspective that takes into account both empirically based and classroom-oriented research from cognitive and developmental views of WNA. Classroom intervention studies do not easily permit generalisation, nor do they reflect the highly controlled experimental settings of the neuro-cognitive studies, but these studies are critical to informing further research, mathematics teaching practice and curriculum development.

Several chapters in the volume have centred on teaching practices and tools for learning whole number arithmetic. For example, working group 3 (Chap. 9) discusses cultural artefacts and tasks and working group 4 (Chap. 11) teaching and assessment approaches. While this chapter has delved into the cognitive and neurocognitive bases of research related to concept development in number, there are clearly synergies between this chapter and teaching approaches.

Some important messages emanating from the working groups have been articulated for teachers so that they develop their professional knowledge and improved awareness of the complexities of whole number learning. Cognitive and neurocognitive approaches can enable new insights to be incorporated into teaching practices. Aligned with new insights is the need for effective professional learning programmes to enable teachers to implement and review new approaches, tasks or assessment practices that they adopt. Developing a better understanding of the wide variations in students' strategies and the difficulties students experience in acquisition of number concepts is critical to improving mathematics learning overall. 
The research discussed in this chapter can provide to some extent explanations and possible interventions to assist teachers to focus on core mathematical foundations such as numerical magnitude representation and the mental number line, structures and relationships in developing number sense, promoting multiplicative thinking rather than restricting focus on counting and additive computations and attention to the role of spatial origins of number learning. The role of students' representations and interpretations of those representations has been exemplified. These examples may assist teachers in selecting appropriate representational tools and tasks to promote better understanding of whole number relationships. In conclusion this chapter has raised new questions from a range of perspectives, both neuro-cognitive and cognitive, but with a common goal of providing new insights into the complex and dynamic nature of young students' whole number learning.

\section{References}

Aunio, P., \& Niemivirta, M. (2010). Predicting children's mathematical performance in grade one by early numeracy. Learning and Individual Differences, 20, 427-435.

Baccaglini-Frank, A., \& Bartolini Bussi, M. G. (2015). Buone pratiche didattiche per prevenire falsi positivi nelle diagnosi di discalculia: Il progetto PerContare.Form@re-Open Journal per la formazione in rete, 15(3), 170-184.

Baccaglini-Frank, A., \& Maracci, M. (2015). Multi-touch technology and preschoolers' development of number-sense. Digital Experiences in Mathematics Education, 1(1), 7-27.

Baccaglini-Frank, A., \& Scorza, M. (2013). Preventing learning difficulties in early arithmetic: The PerContare Project. In T. Ramiro-Sànchez \& M. P. Bermùdez (Eds.), Libro de Actas I Congreso Internacional de Ciencias de la Educatiòn y des Desarrollo (p. 341). Granada: Universidad de Granada.

Baroody, A. J. (1999). Children's relational knowledge of addition and subtraction. Cognition and Instruction, 17(2), 137-175.

Baroody, A. J., Torbeyns, J., \& Verschaffel, L. (2009). Young children's understanding and application of subtraction-related principles. Mathematical Thinking and Learning, 11(1-2), 2-9.

Bartolini Bussi, M. G., \& Mariotti, M. A. (2008). Semiotic mediation in the mathematics classroom artefacts and signs after a Vygotskian perspective. In L. English (Ed.), Handbook of international research in mathematics education (2nd ed., pp. 746-783). New York: Routledge.

Beck, D. M. (2010). The appeal of the brain in the popular press. Perspectives on Psychological Science, 5, 762-766.

Booth, J. L., \& Siegler, R. S. (2008). Numerical magnitude representations influence arithmetic learning. Child Development, 79, 1016-1031.

Britt, M. S., \& Irwin, K. C. (2011). Algebraic thinking with and without algebraic representation: A pathway for learning. In J. Cai \& E. Knuth (Eds.), Early algebraization: A global dialogue from multiple perspectives (pp. 137-160). Berlin: Springer.

Bryant, P., Christie, C., \& Rendu, A. (1999). Children's understanding of the relation between addition and subtraction: Inversion, identity and decomposition. Journal of Experimental Child Psychology, 74, 194-212.

Butterworth, B. (2005). The development of arithmetical abilities. Journal of Child Psychology and Psychiatry, 46(1), 3-18.

Butterworth, B., \& Reeve, R. (2008). Verbal counting and spatial strategies in numerical tasks: Evidence from indigenous Australia. Philosophical Psychology, 21, 443-457.

Clayton, S., \& Gilmore, C. (2015). Inhibition in dot comparison tasks. ZDM Mathematics Education, 47(5), 759-770. 
Clements, D. H., \& Sarama, J. (2011). Tools for early assessment in math (TEAM). Teacher's Guide. Columbus: McGraw-Hill Education Series.

Coles, A. (2014). Ordinality, neuro-science and the early learning of number. In P. Liljedahl, C. Nicol, S. Oesterle, \& D. Allan (Eds.), Proceedings of the Thirty-eighth annual conference of the International Group for the Psychology of Mathematics Education (Vol. 2, pp. 329-336). Vancouver: PME.

De Smedt, B., Ansari, D., Grabner, R. H., Hannula-Sormunen, M., Schneider, M., \& Verschaffel, L. (2011). Cognitive neuroscience meets mathematics education: It takes two to tango. Educational Research Review, 6, 232-237.

Dehaene, S. (2011). The number sense: How the mind creates mathematics. New York: Oxford University Press.

Dehaene, S., Piazza, M., Pinel, P., \& Cohen, L. (2003). Three parietal circuits for number processing. Cognitive Neuropsychology, 20, 487-506.

Dowker, A. (2008). Individual differences in numerical abilities in preschoolers. Developmental Science, 11, 650-654.

Ensor, P., Hoadley, U., Jacklin, H., Kuhn, C., Schnitte, E., Lombard, A., \& van den HeuvelPanhuizen, M. (2009). Specialising pedagogical text and time in Foundation Phase numeracy classrooms. Journal of Education, 47, 5-30.

Fuson, K. C. (1988). Children's counting and concepts of number. New York: Springer-Verlag.

Gattegno, C. (1974). The common sense of teaching mathematics. New York: Educational Solutions Worldwide Inc..

Geary, D. C., Bow-Thomas, C. C., \& Yao, Y. (1992). Counting knowledge and skill in cognitive addition: A comparison of normal and mathematically disabled children. Journal of Experimental Child Psychology, 54, 372-391.

Gebuis, T., \& Reynvoet, B. (2012). The interplay between nonsymbolic number and its continuous visual properties. Journal of Experimental Psychology: General, 14, 642-648.

Gilmore, C., \& Bryant, P. (2006). Individual differences in children's understanding of inversion and arithmetical skill. British Journal of Educational Psychology, 76, 309-331.

Gilmore, C., Attridge, N., \& Inglis, M. (2011). Measuring the approximate number system. Quarterly Journal of Experimental Psychology, 64, 2099-2109.

Göbel, S. M., Watson, S. E., Lervåg, A., \& Hulme, C. (2014). Children's arithmetic development: It is number knowledge, not the approximate number sense, that counts. Psychological Science, 25(3), 789-798.

Griffin, S. (2004). Teaching number sense. Educational Leadership, 61(5), 39-42.

Hannula, M. M., \& Lehtinen, E. (2005). Spontaneous focusing on numerosity and mathematical skills of young children. Learning and Instruction, 15(3), 237-256.

Jordan, N. C., Glutting, J., \& Ramineni, C. (2010). The importance of number sense to mathematics achievement in first and third grades. Learning and Individual Differences, 20, 82-88.

Krajewski, K., \& Schneider, W. (2009). Early development of quantity to number-word linkage as a precursor of mathematical school achievement and mathematical difficulties: Findings from a four-year longitudinal study. Learning and Instruction, 19(6), 513-526.

Lyons, I., \& Beilock, S. (2011). Numerical ordering ability mediates the relation between numbersense and arithmetic competence. Cognition, 121(2), 256-261.

McMullen, J., Hannula-Sormunen, M. M., \& Lehtinen, E. (2013). Young children's recognition of quantitative relations in mathematically unspecified settings. Journal of Mathematical Behavior, 32, 450-460.

McMullen, J., Hannula-Sormunen, M. M., \& Lehtinen, E. (2014). Spontaneous focusing on quantitative relations in the development of children's fraction knowledge. Cognition and Instruction, 32(2), 198-218. 
Mulligan, J. T., \& Mitchelmore, M. C. (2009). Awareness of pattern and structure in early mathematical development. Mathematics Education Research Journal, 21(2), 33-49.

Mulligan, J. T., \& Mitchelmore, M. C. (2013). Early awareness of mathematical pattern and structure. In L. English \& J. Mulligan (Eds.), Reconceptualizing early mathematics learning (pp. 29-46). Dordrecht: Springer.

Mulligan, J. T., Mitchelmore, M. C., English, L., \& Crevensten, N. (2013). Reconceptualizing early mathematics learning: The fundamental role of pattern and structure. In L. English \& J. Mulligan (Eds.), Reconceptualizing early mathematics learning (pp. 47-66). Dordrecht: Springer.

Mulligan, J. T., Mitchelmore, M. C., \& Stephanou, A. (2015). Pattern and Structure Assessment (PASA): An assessment program for early mathematics (Years $F-2$ ) teacher guide. Melbourne: ACER Press.

Nunes, T., Bryant, P., Burman, D., Bell, D., Evans, D., \& Hallett, D. (2008). Deaf children's informal knowledge of multiplicative reasoning. Journal of Deaf Studies and Deaf Education, 14, 260-277.

Nunes, T., Bryant, P., Barros, R., \& Sylva, K. (2012). The relative importance of two different mathematical abilities to mathematical achievement. British Journal of Educational Psychology, 82, 136-156.

Peeters, D., Degrande, T., Ebersbach, M., Verschaffel, L., \& Luwel, K. (2015). Children's use of number line estimation strategies. European Journal of Psychology of Education. (Online first) doi:https://doi.org/10.1007/s10212-015-0251-z

Peters, G., De Smedt, B., Torbeyns, J., Ghesquière, P., \& Verschaffel, L. (2010). Using addition to solve subtractions in the number domain up to 20. Acta Psychologica, 133, 163-169.

Piaget, J., \& Szeminska, A. (1952). The child's conception of number. London: Routledge. (Original work published 1941).

Reeve, R., Reynolds, F., Humberstone, J., \& Butterworth, B. (2012). Stability and change in markers of core numerical competencies. Journal of Experimental Psychology: General, 141, 649-666.

Resnick, L. B., Bill, V. L., Lesgold, S. B., \& Leer, N. M. (1991). Thinking in arithmetic class. In B. Means, C. Chelemer, \& M. S. Knapp (Eds.), Teaching advanced skills to at-risk students (pp. 27-53). San Francisco: Jossey-Bass.

Robinson, K. M., Ninowski, L. E., \& Gray, M. L. (2006). Children's understanding of the arithmetic concepts of inversion and associativity. Journal of Experimental Child Psychology, 94, 349-362.

Rubinsten, O., \& Henik, A. (2005). Automatic activation of internal magnitudes: A study of developmental dyscalculia. Neuropsychology, 19, 641-648.

Sasanguie, D., \& Reynvoet, B. (2013). Number comparison and number line estimation rely on different mechanisms. Psychologica Belgica, 53, 17-35.

Sasanguie, D., Defever, E., Maertens, B., \& Reynvoet, B. (2014). The approximate number system is not predictive for symbolic number processing in kindergarteners. The Quarterly Journal of Experimental Psychology, 67, 271-280.

Schleifer, P., \& Landerl, K. (2011). Subitizing and counting in typical and atypical development. Developmental Science, 14, 280-291.

Schmittau, J. (2011). The role of theoretical analysis in developing algebraic thinking: A Vygotskian perspective. In J. Cai \& E. Knuth (Eds.), Early algebraization: A global dialogue from multiple perspectives (pp. 71-86). Berlin: Springer.

Schneider, M., Beeres, K., Coban, L., Merz, S., Schmidt, S. S., Stricker, J., \& De Smedt, B. (2017). Associations of non-symbolic and symbolic numerical magnitude processing with mathematical competence: A meta-analysis. Developmental Science, 20(3), p. 16.

Schoenfeld, A. H. (2007). Method. In F. K. Lester (Ed.), Second handbook of research on mathematics teaching and learning (pp. 69-107). Charlotte: Information Age Publishing. 
Siegler, R. S., \& Booth, J. L. (2004). Development of numerical estimation in young children. Child Development, 75, 428-444.

Siegler, R. S., \& Lemaire, P. (1997). Older and younger adults' strategy choices in multiplication: Testing predictions of ASCM using the choice/no-choice method. Journal of Experimental Psychology: General, 126, 71-92.

Sinclair, N., \& Jackiw, N. (2011). TouchCounts. Application for the iPad. https://itunes.apple.com/ ca/app/touchcounts/id897302197?mt=8??

Sinclair, N., \& Pimm, D. (2015). Whatever be their number: Counting on the visible, the audible, and the tangible. In M. Meletiou-Mavrotheris, K. Mavrou, \& E. Paparistodemou (Eds.), Integrating touch-enabled and mobile devices into contemporary mathematics education (pp. 50-80). Hershey: IGI Global.

Skwarchuk, S. L., Sowinski, C., \& LeFevre, J. A. (2014). Formal and informal home learning activities in relation to children's early numeracy and literacy skills: The development of a home numeracy model. Journal of Experimental Child Psychology, 121, 63-84.

Stokes, D. E. (1997). Pasteur's quadrant: Basic science and technical innovation. Washington, DC: Brookings.

Watson, A., \& Ohtani, M. (2015). Task design in mathematics education: The 22nd ICMI Study. New York: Springer.

\section{Cited papers from Sun, X., Kaur, B., \& Novotna, J. (Eds.). (2015). Conference proceedings of the ICMI study 23: Primary mathematics study on whole numbers. Retrieved February 10, 2016, from www.umac.mo/fed/ICMI23/doc/Proceedings_ICMI_ STUDY_23_final.pdf}

Baccaglini-Frank, A. (2015). Preventing low achievement in arithmetic through the didactical materials of the PerContare project (pp. 169-176).

Butterworth, B. (2015). Low numeracy: From brain to education (pp. 21-33).

Elia, I., \& van den Heuvel-Panhuizen, M. (2015). Mapping kindergartners' number competence (pp. 177-185).

Gervasoni, A., \& Parish, L. (2015). Insights and implications about the whole number knowledge of grade 1 to grade 4 children (pp. 440-447).

Gould, P. (2015). Recalling a number line to identify numerals (pp. 186-193).

He, S. (2015). How do Chinese students solve addition/subtraction problems: A review of cognitive strategy (pp. 194-202).

Ma, Y., Xie, S., \& Wang, Y. (2015). Analysis of students' systematic errors and teaching strategies for 3-digit multiplication (pp. 203-211).

Milinković, J. (2015). Counting strategies and system of natural number representations in young children (pp. 212-219).

Mulligan, J., \& Woolcott, G. (2015). What lies beneath? Conceptual connectivity underlying whole number arithmetic (pp. 220-228).

Obersteiner, A., Moll, G., Reiss, K., \& Pant, H. A. (2015). Whole number arithmetic: Competency models and individual development (pp. 245-250).

Roberts, N. (2015). Interpreting children's representations of whole number additive relations in the early grades (pp. 245-250).

Sayers, J., \& Andrews, P. (2015). Foundational number sense: The basis for whole number arithmetic competence (pp. 124-131).

Sinclair, N., \& Coles, A. (2015). 'A trillion is after one hundred': Early number and the development of symbolic awareness (pp. 251-259). 
Verschaffel, L., Torbeyns, J., Peters, G., De Smedt, B., \& Ghesquière, P. (2015). Analysing subtraction-by-addition in the number domain $20-100$ by means of verbal protocol vs reaction time data (pp. 260-267).

Yang, D-C. (2015). Performance of fourth graders in judging reasonableness of computational results for whole numbers (pp. 268-276).

Young-Loveridge, J., \& Bicknell, B. (2015). Using multiplication and division contexts to build place-value understanding (pp. 379-387).

Open Access This chapter is licensed under the terms of the Creative Commons Attribution 4.0 International License (http://creativecommons.org/licenses/by/4.0/), which permits use, sharing, adaptation, distribution and reproduction in any medium or format, as long as you give appropriate credit to the original author(s) and the source, provide a link to the Creative Commons license and indicate if changes were made.

The images or other third party material in this chapter are included in the chapter's Creative Commons license, unless indicated otherwise in a credit line to the material. If material is not included in the chapter's Creative Commons license and your intended use is not permitted by statutory regulation or exceeds the permitted use, you will need to obtain permission directly from the copyright holder.

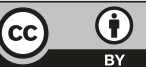

